\title{
Evaluation of Milk Quality by Ultrasonic Technique
}

\author{
Chien-Hsing Chen ${ }^{*}$ and Li-De Zhang \\ Department of Information Technology, Meiho University, \\ 23, Pingguang Rd., Neipu, Pingtung, Taiwan \\ (Received May 31, 2018; accepted October 18, 2018)
}

Keywords: mastitis, ultrasound, California mastitis test

A high-frequency $(1 \mathrm{MHz})$ ultrasonic device was applied to investigate the relationships between the somatic cell count (SCC) in milk and the physical properties measured through the changes in the velocity and attenuation of wave propagation during gelation formation in the California mastitis test (CMT). Owing to nonhomogenous mixing without shear applied in CMT gelation, ultrasonic signals were more unstable than those under a complete mixing condition. On the basis of the complete mixing condition, the velocity had no significant relationship with SCC concentration; however, there was a clearly positive relationship between attenuation and SCC concentration. In addition, the linear fitting slopes of attenuation during the measurement were positive as the SCC concentration was less than the threshold value $\left(\mathrm{SCC}=5 \times 10^{4}\right.$ cells $\left./ \mathrm{ml}\right)$. In contrast, the slopes became negative.

\section{Introduction}

Mastitis is undoubtedly one of the most serious and costly infectious diseases affecting dairy cow production. ${ }^{(1)}$ Although the exact cost is difficult to quantify, it was estimated in the USA to be $\$ 2$ billion in 2009. ${ }^{(2)}$ Obviously, an early diagnosis for mastitis would improve the management of mastitis and may lead to possible reduction in these losses. One of the obvious symptoms of mastitis is the increase in milk somatic cell count (SCC), which includes epithelial and white blood cells. Somatic cells are induced by the cow's immune system to fight infection-causing bacteria. Therefore, the ideal solution would be a sensor that directly measures SCC automatically on-line. The measured SCC in raw milk is widely accepted as the most useful indirect indicator of mastitis and milk quality. However, SCC is determined in laboratories by flow cytometry. To achieve a faster, but less accurate result, some farmers use a simple, inexpensive, cow-side test, known as the California mastitis test (CMT) ${ }^{(3)}$ to provide a qualitative estimate of SCC in the foremilk of individual cows or quarters.

CMT is an indirect method of detecting infected quarters on the farm on the basis of the reaction of special detergents with the DNA of somatic cells and the increase in the viscosity of the mixture, which is proportional to SCC, and the degree of gel formation is evaluated by gently rotating the CMT paddle. However, the test involves a subjective evaluation of the degree of gelling reaction between the milk sample and the reagent, which might result in false *Corresponding author: e-mail: jameschen@meiho.edu.tw https://doi.org/10.18494/SAM.2019.2097 
positives and negatives. Also, the relationship between the subjective rating and SCC in the milk sample (an indicator of the degree of udder infection) is not sufficiently accurate for many farmers.

Currently, farmers have access to indirect estimates of SCC, such as in-line conductivity, but not to automated on-line sensors that directly measure SCC. The mechanization or standardization of CMT has been attempted using rotary viscometers, ${ }^{(4,5)}$ falling ball viscometers, ${ }^{(6)}$ a rolling ball viscometer ${ }^{(7)}$ or some measurements based on the flow of CMT gel through holes or tubes of various diameters, such as the Wisconsin mastitis test ${ }^{(8)}$ and Brabant mastitis reaction. ${ }^{(9,10)}$ However, none of these systems were practically suitable for on-line use because some time is required to conduct the test, which reduces parlor throughput.

Fast analysis devices or systems are highly desirable as they would allow an on-line response to the changes in medium components. However, most on-line systems currently used have a low accuracy. The gel has been described as thixotropic, ${ }^{(7,11)}$ that is, the gel breaks down under shear force, but it reforms with the removal of the force. Also, the rheology of the gel was investigated by both capillary and rotational viscometry, ${ }^{(12)}$ and it was found that the gel was non-Newtonian, but the initial phase of viscosity increase was not due to shear dependence, but rather to the gelation reaction. It can therefore be deduced that the rheology of the gel is complex not only because it is non-Newtonian, but also because of the strong dependence on test conditions. These make designing a successful sensor much more challenging.

With this background, ultrasound becomes an alternative technique for the on-line monitoring of mastitis by CMT. The most important features of ultrasonic systems are robustness, noninvasiveness, precision, low cost, rapidity, and easy automation. Furthermore, ultrasound can be used to analyze opaque materials, offering an alternative to electromagneticwave-based devices. Recently, on-line ultrasonic techniques have been used for monitoring alcoholic fermentation, ${ }^{(13,14)}$ dairy fermentation, ${ }^{(15)}$ dough fermentation, ${ }^{(16)}$ Escherichia coli growth, ${ }^{(17,18)}$ and fat globule size distribution selection. ${ }^{(19,20)}$

Understanding the ultrasonic properties of the CMT gel is important in the design and operation of an automated CMT that yields a qualitative estimate of SCC. This study is conducted to investigate the ultrasonic characteristics of CMT gel formation and to determine whether a reliable and inexpensive automated on-line SCC detector based on CMT can be developed. Therefore, measurements of ultrasonic velocity and attenuation in the CMT gel have been carried out and used to understand ultrasonic velocity and attenuation changes.

\section{Materials and Methods}

An acquired ultrasonic waveform (4 cycles) is shown in Fig. 1. The first pulse wave, peak $1 \mathrm{a}$, is the input wave and the second pulse wave, peak $2 \mathrm{a}$, is the output wave. Then, the next incoming wave is the input wave obtained from the first pulse wave in Fig. 1. From the waveform signal data, the ultrasonic velocity $(c, \mathrm{~m} / \mathrm{s})$ and attenuation $(\alpha$, neper/m) of the CMT gel were calculated as follows: ${ }^{(21)}$

$$
c=8 d / t_{r}
$$




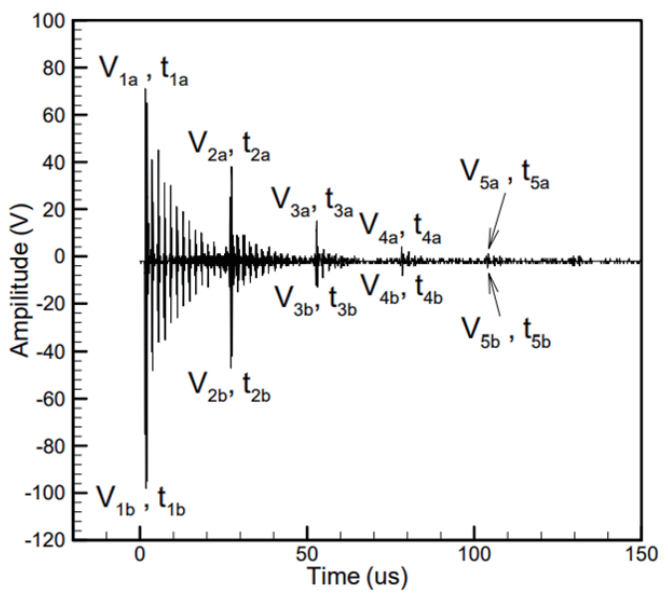

Fig. 1. Waveform of ultrasonic pulse travelling through the sample.

$$
\alpha=[1 /(2 d)] \ln \Delta V,
$$

where $t_{r}=\left[\left(t_{5 a}-t_{1 a}\right)+\left(t_{5 b}-t_{1 b}\right)\right], d=$ distance traversed by the ultrasonic pulse (m), and

$$
\Delta V=\frac{1}{8}\left[\frac{V_{1 b}}{V_{2 b}}+\frac{V_{2 b}}{V_{3 b}}+\frac{V_{3 b}}{V_{4 b}}+\frac{V_{4 b}}{V_{5 b}}+\frac{V_{1 a}}{V_{2 a}}+\frac{V_{2 a}}{V_{3 a}}+\frac{V_{3 a}}{V_{4 a}}+\frac{V_{4 a}}{V_{5 a}}\right],
$$

with $V_{1 a}, V_{1 b}$, and so forth being the signal amplitudes $(\mathrm{V})$, and $t_{1 a}, t_{1 b}$, and so forth being the time (s) corresponding to different peaks in Fig. 1.

An ultrasonic pulse echo experimental setup was used. It is composed of one 1-MHzcentral-frequency ultrasonic longitudinal wave transducer (Model P/N V314, Panametrics), an ultrasonic pulser receiver (Model 5072PR, Panametrics), a digital storage oscilloscope (Model GDS1000, GW Instek), and a computer equipped with analysis software to obtain the time of flight, which is the time taken by the ultrasonic pulse to travel through the sample.

The transducer was mounted on one side of the circular cross section of a cylinder sample tank submerged in a water bath at $30 \pm 0.1{ }^{\circ} \mathrm{C}$ in order to minimize the effect of temperature on the measurements. The traversing distance between the transducer and the reflective surface of the cylinder was $1.9 \mathrm{~cm}$.

Foremilk $(50 \mathrm{ml})$ was taken from each calf to determine SCC at the Testing Laboratory, Hsinchu Branch, Livestock Research Institute, Council of Agriculture, Taiwan. On the basis of the SCC, udders were classified into three groups, namely, healthy udders (below $50 \times 10^{4}$ cells $/ \mathrm{ml})$, minor-infection udders $\left(50 \times 10^{4}-100 \times 10^{4}\right.$ cells $\left./ \mathrm{ml}\right)$, and seriously infected udders (above $100 \times 10^{4}$ cells $/ \mathrm{ml}$ ) (International Dairy Federation, 1987). Therefore, the SCC threshold used in this study was $50 \times 10^{4}$ cells $/ \mathrm{ml}$. Raw milk $(6 \mathrm{ml})$ was combined with an anionic surfactant at a ratio of 1:1 (SDS: sodium dodecyl sulfate, $0.1 \mathrm{~g} / \mathrm{ml}$, in distilled water). All experiments were replicated three times. 


\section{Results and Discussion}

To investigate the ultrasonic characteristics of CMT milk during gel reaction, there were two different states of CMT gel mixing to be measured. One was incomplete mixing, in which the signals began to be measured while the raw milk was combined with an equal amount of SDS. The other was complete mixing, in which the mixture was mixed by manual rotation for $60 \mathrm{~s}$ and then measured, reducing the effect of gel reaction on ultrasonic propagation. The ultrasonic velocity and attenuation in CMT milk gel for incomplete and complete mixing processes were estimated at $\mathrm{SCC}=23.3426\left(10^{3} \mathrm{cells} / \mathrm{ml}\right), T=30^{\circ} \mathrm{C}$, and $f=1 \mathrm{MHz}$, as shown in Figs. 2 and 3, respectively. The dashed lines indicate the results of linear fitting of the experimental data.

For incomplete mixing, the velocity significantly increased with time for $150 \mathrm{~s}$, which was induced by the clear change in viscosity in the initial state. Then, it slightly decreased and became constant. Nevertheless, the velocity for complete mixing did not clearly change during the measurement. In addition, the attenuation for incomplete mixing increased with time for $120 \mathrm{~s}$. Then, it significantly decreased and became constant after $200 \mathrm{~s}$ and the same as that observed for complete mixing. However, the attenuation for complete mixing slightly increased with time during the measurement.

According to the results described above, the signal for incomplete mixing was less stable than that for complete mixing. The main reason for the incomplete mixing was that CMT milk was a nonhomogeneous mixture that was mixed by the diffusion effect without any application of shear force. Therefore, the ultrasonic velocity and attenuation for CMT milk were sensitive to the degree of mixing. These trends were expected when the signals were more stable under a complete mixing condition. In addition, to obtain valid quantitative CMT scores using ultrasonic technology, the effect of the degree of mixing should be reduced. Therefore, measurement should be performed under a complete mixing condition.

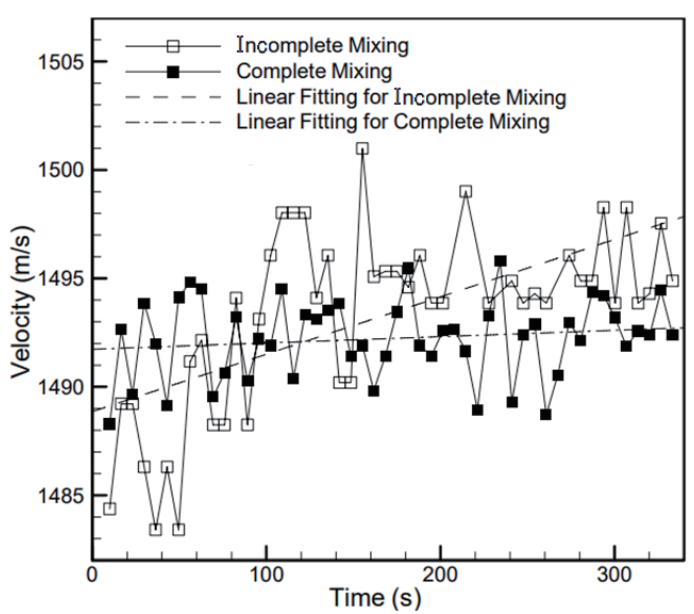

Fig. 2. Ultrasonic velocities in CMT milk for incomplete and complete mixing processes. $\mathrm{SCC}=$ $23.3426\left(10^{3}\right.$ cells $\left./ \mathrm{ml}\right), T=30^{\circ} \mathrm{C}$, and $f=1 \mathrm{MHz}$.

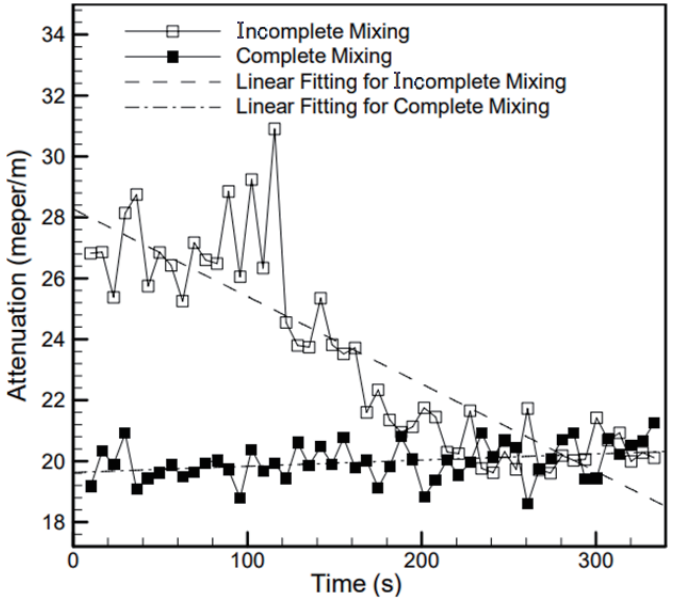

Fig. 3. Ultrasonic attenuations of CMT milk for incomplete and complete mixing processes. $\mathrm{SCC}=$ $23.3426\left(10^{3} \mathrm{cells} / \mathrm{ml}\right), T=30^{\circ} \mathrm{C}$, and $f=1 \mathrm{MHz}$. 
Because the viscosity of the CMT gel is related to SCC concentration, the relationships of velocity and attenuation with SCC concentration were compared with the SCC threshold $(50 \times$ $10^{4}$ cells $/ \mathrm{ml}$ ) under a complete mixing condition, as shown in Figs. 4 and 5, respectively. The comparison revealed that there was a nonsignificant relationship between velocity and SCC concentration, as shown in Fig. 4. In addition, the results in Fig. 5 indicated that the ultrasonic attenuation had a good positive relationship with SCC concentration, probably because the friction of ultrasound propagating in gelation was clearly affected by the gel viscosity, which may allow some ultrasonic energy to be adsorbed and turned into heat.

Referring to the results for the complete mixing condition shown in Fig. 3, the slopes of the linear fitted curves of attenuation under different SCC concentrations were compared, as shown in Fig. 6. The results indicated that, when SCC was less than the threshold, the linear fitted slope of attenuation was positive. In contrast, it became negative when SCC was greater

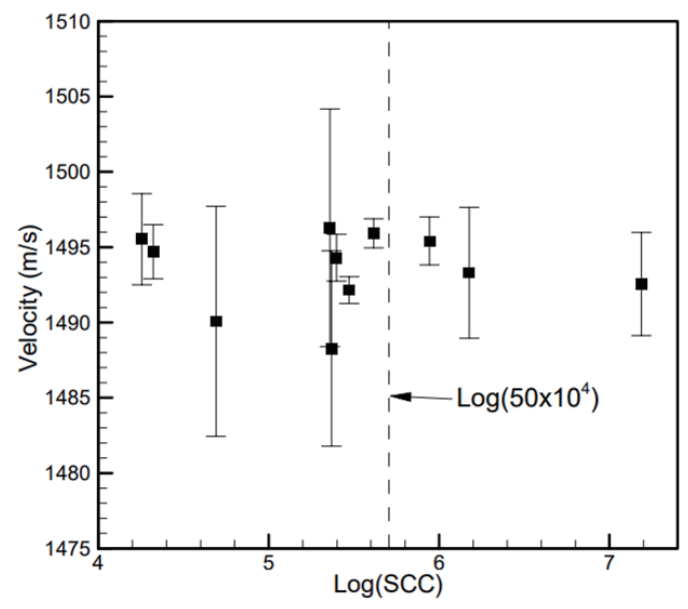

Fig. 4. Relationship between velocity and $\log (\mathrm{SCC})$ with the threshold (dashed line) under a complete mixing condition.

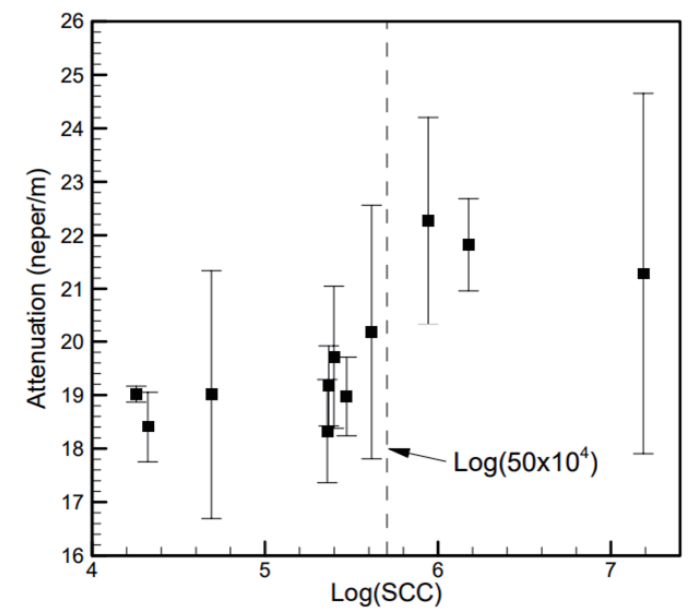

Fig. 5. Relationship between attenuation and $\log (\mathrm{SCC})$ under a complete mixing condition.

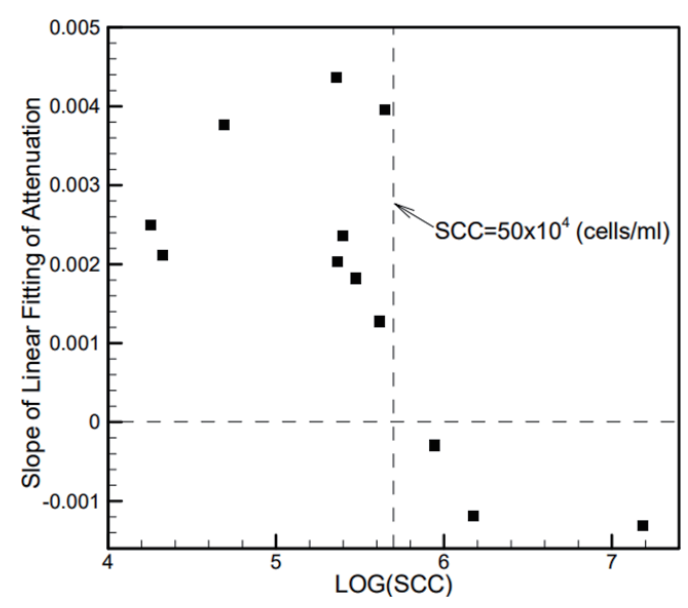

Fig. 6. Relationship between slope of linear fitting of attenuation and $\log (\mathrm{SCC})$ during the measurement under a complete mixing condition. 
than the threshold. On the basis of the results described above, the evaluation of attenuation in CMT using ultrasonic technology is promising as an alternative method of detecting mastitis. Although the detection limit of CMT scores was SCC $=50 \times 10^{4}$ cells $/ \mathrm{ml}$, a more automatic mixing procedure would improve the accuracy of CMT scores in the future.

\section{Conclusions}

In this study, a method of investigating CMT gelation using ultrasonic technology was evaluated. Under incomplete and complete mixing conditions, the physical properties of the velocity and attenuation of wave propagation during gelation formation in CMT were presented. Ultrasonic signals were more unstable for nonhomogenous mixing than for complete mixing. With the complete mixing condition used as the reference, the velocity had no significant relationship with SCC concentration, but there was a clearly positive relationship between attenuation and SCC concentration. In addition, as the SCC concentration was less than the threshold $\left(\mathrm{SCC}=50 \times 10^{4}\right.$ cells $/ \mathrm{ml}$ ), the slopes of linear fitting attenuation during the measurement were positive. In contrast, the slopes became negative under the complete mixing condition. Therefore, the relationship between attenuation and SCC concentration could be used to evaluate the CMT scores by this ultrasonic technology. However, there is a need to further investigate the relationship between SDS concentration and SCC threshold to improve the SCC scores obtained by ultrasonic technology.

\section{Acknowledgments}

The authors would like to thank the Ministry of Science and Technology of Taiwan for financially supporting this research under Contract No. MOST 105-2221-E-276-001.

\section{References}

1 B. Heringstad, G. Klemetsdal, and J. Ruane: Livest. Prod. Sci. 64 (2000) 95.

2 C. Viguier, S. Arora, N. Gilmartin, K. Welbeck, and R. O’Kennedy: Trends Biotechnol. 27 (2009) 486.

3 O. W. Schalm and D. Noorlander: J. Am. Vet. Med. Assoc. 130 (1957) 199.

4 G. Nageswarao and H. E. Calbert: J. Milk Food Technol. 32 (1969) 365.

5 M. G. Nichols and D. S. M. Phillips: Aust. J. Dairy Technol. 27 (1972) 134.

6 X. Carre: Thesis, Ecole Nationale Veterinaire Lyon (1970).

7 W. Whittlestone, I. Fell, and H. De Langen: J. Milk Food Technol. 33 (1970) 351.

8 D. I. Thompson and D. S. Postle: J. Milk Food Technol. 27 (1964) 271

9 F. H. J. Jaartsveld: Neth. Milk Dairy J. 16 (1962) 260.

10 V. Bottazzi: IL Latte 37 (1963) 755.

11 W. Whittlestone and I. Fell: Proc. 36th Ann. Conf. New Zealand Dairy Sci. Assoc. (1965) 21.

12 C. J. Verbeek, S. S. Xia, and D. Whyte: J. Dairy Res. 75 (2008) 385.

13 T. Becker, M. Mitzscherling, and A. Delgado: Eng. Life Sci. 1 (2001) 61.

14 P. Resa, L. Elvira, and F. Montero de Espinosa: Food Res. Int. 37 (2004) 587.

15 L. Elvira, P. Resa, and F. Montero de Espinosa: Proc. Forum Acusticum (2002).

16 H. M. Elmehdi, J. H. Page, and M. G. Scanlon: Food Bioprod. Process. 81 (2003b) 217.

17 B. R. Reddy, Y. H. Babu, and D. L. Reddy: Mater. Lett. 49 (2001) 47.

18 C. Sierra, L. Elvira, J. L. García, P. Resa, and B. Galán: Appl. Microbiol. Biotechnol. 85 (2010) 813.

19 S. Karlović, T. Bosiljkov, M. Brnčić, D. Semenski, F. Dujmić, B. Tripalo, and D. Ježeka: Chem. Biochem. Eng. Q 28 (2014) 499.

20 S. P. Itle and D. R. Olver: J. Anim. Sci. 94 (2016) 19.

21 A. Chyung and S. Gunasekaran: J. Food Eng. 58 (2003) 103. 


\section{About the Authors}

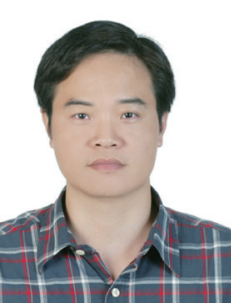

Chien-Hsing Chen received his M.E. and Ph.D. degrees in Mechanical Engineering from National Cheng-Chung University, Taiwan, in 1997 and 2006, respectively. Since then, he has been with the Information Technology Department of Meiho University, Taiwan, where he is currently an assistant professor. His research interests are in robotics, IoT, and sensors.

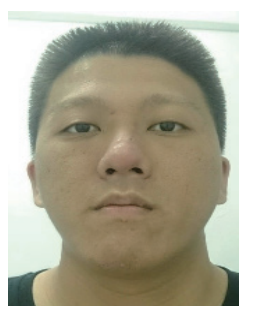

Li-De Zhang is an undergraduate student of the Information Technology Department of Meiho University, Taiwan. 